\title{
Cell Cycle Arrest and Apoptosis Induced by Kinamycin F in Human Osteosarcoma Cells
}

\author{
ALBERTO BAVELLONI ${ }^{1}$, ENRICO FOCACCIA ${ }^{1,2}$, MANUELA PIAZZI ${ }^{1,2}$, \\ COSTANTINO ERRANI ${ }^{3}$, WILLIAM BLALOCK ${ }^{1,2^{*}}$ and IRENE FAENZA ${ }^{4 *}$ \\ ${ }^{1}$ Laboratory of Musculoskeletal Cell Biology, Rizzoli Orthopedic Institute, Bologna, Italy; \\ ${ }^{2}$ National Research Council of Italy (CNR) - Institute of Molecular Genetics (IGM), UOS-Bologna, Bologna, Italy; \\ ${ }^{3}$ Orthopaedic Oncology Surgical Unit, Rizzoli Orthopedic Institute, Bologna, Italy; \\ ${ }^{4}$ Cellular Signaling Laboratory, Department of Biomedical Sciences, University of Bologna, Bologna, Italy
}

\begin{abstract}
Background/Aim: Kinamycin $F$ is a bacterial metabolite which contains an unusual and potentially reactive diazo group that is known for its ability to inhibit cell growth. In this study, the potential anti-tumor activity of kinamycin $F$ was investigated in three human osteosarcoma cell lines, MG-63, U-2 OS and HOS as an antitumor agent with a potentially novel target. Materials and Methods: Proliferation and cell viability were measured in three human osteosarcoma cell lines by commercially available kits. We also evaluated the effects of the drug on cell cycle progression using the Muse ${ }^{T M}$ Cell Analyzer. Caspase-3 activity was determined by a fluorometric EnzChek assay kit. Finally, following treatment with kinamycin $F$ the protein levels of cyclin D3, cyclin $A$ and $c d K-2$ were examined. Results: Kinamycin $F$ induced a concentration-dependent cell death in all the three cell lines. Flow cytometry revealed that kinamycin $F$ treatment at $1 \mu \mathrm{M}$ concentration significantly increased the cell population in the $G_{2} / M$-phase (60-65\%). Kinamycin $F$ activated caspase 3 in all the three cell lines, clearly demonstrating that the growth inhibitory effect of kinamycin $F$ can be attributed to apoptosis induction. Finally, kinamycin $F$ suppressed osteosarcoma cell proliferation affecting cyclin A and D3 expression. Conclusion: Understanding the mechanism by which
\end{abstract}

\footnotetext{
*These Authors equally contributed to this work.

Correspondence to: Irene Faenza, Cellular Signaling Laboratory, Department of Biomedical Sciences, University of Bologna, Bologna, Italy. Tel: +393392287797, e-mail: irene.faenza2@unibo.it; and William Blalock, National Research Council of Italy (CNR)-Institute of Molecular Genetics (IGM), UOS-Bologna, Bologna, Italy. Tel: +39 051626770,e-mail: william.blalock@cnr.it
}

Key Words: Osteosarcomas, cyclin D3, cell death, kinamycin F. kinamycin $F$ exerts its ability to inhibit cell growth may be a step forward in the development of new therapeutic strategies for the treatment of OS.

Osteosarcoma is an aggressive bone tumor characterized by malignant osteoid production and malignant cells with osteoblastic differentiation. It is the primary bone tumor mainly in young adults and children (1). This tumor is most often located in the proximal tibia, the proximal humerus, and the distal femur. Although progresses in therapeutic strategies including radiotherapy, adjuvant chemotherapy, and wide tumor excision have been achieved, it remains the second leading cause of tumor-related deaths in young adults (2). The disease-free survival and 5-year survival rates are still unsatisfactory (2). Therefore, it is of great importance to understand the molecular mechanisms underlying the initiation, development, and metastasis of osteosarcoma and its response to therapy (3). A variety of agents have been investigated for the treatment of osteosarcoma in clinical trials. In the last few years a great effort has been made to develop new therapeutic strategies for OS patients. Several studies and clinical trials have been testing multimodal therapies and dose variations of classical drugs. However, a great majority of these studies fail to enter phase III clinical trials (4). Currently, the primary treatment for osteosarcoma is a combination of surgery and chemotherapy. However, osteosarcoma frequently develops resistance to conventional chemotherapies resulting in tumor recurrence. Amputation of the affected limbs is often the only option but even this usually fails to save a patient's life due to early metastases (5). Identifying the genes and signal transduction pathways responsible for the development and malignant behavior in osteosarcoma will lead to the discovery of new drugs and therapies. In this study, we characterize for the first time the actions of kinamycin $\mathrm{F}$ on MG-63, U-2 OS and HOS human osteosarcoma cells. Kinamycin $\mathrm{F}$ has been recently described as a potent 
differentiating agent of human erythroleukemia cells. In fact, it has been demonstrated that treatment of K562 cells with kinamycin $\mathrm{F}$ induced erythroid differentiation, a rapid apoptotic response, induction of caspase-3/7 activities and a delayed cell cycle progression through the $\mathrm{S}$ and $\mathrm{G}_{2} / \mathrm{M}$ phases (O'Hara et al., 2010). The bacterial metabolite kinamycin $\mathrm{F}$, which is being investigated as a potent antitumor agent, contains an unusual and highly reactive diazo group as well as a paraquinone and a phenol functional group (6). A potential target of kinamycin $F$ is cyclin D3 in that kinamycin $F$ causes a selective reduction of cyclin D3 at the level of transcription. The aim of this study was to explore the effect and the efficacy of kinamycin $\mathrm{F}$ on the proliferation of human osteosarcoma cells. Here, we demonstrate that kinamycin F suppresses osteosarcoma cell growth, and further explore the underlying mechanisms involved.

\section{Materials and Methods}

Materials and cell culture. The sample of kinamycin $\mathrm{F}$ that was used in this study was provided by Professor G. I. Dmitrienko at the University of Waterloo, Waterloo, Ontario, Canada and was prepared semi-synthetically from kinamycin D that was isolated from S. murayamaensis as described previously (7). Control and kinamycin $\mathrm{F}$ treatments were dissolved in DMSO and the final DMSO concentration was always $0.5 \%(\mathrm{v} / \mathrm{v})$. In this study, we analyzed three cell lines derived from human osteosarcomas. The HOS, U-2 OS, and MG-63 human osteosarcoma cell lines were purchased from the American Type Culture Collection (Manassas, VA). Cell lines were routinely cultured in Iscove's modified Dulbecco's medium supplemented with $10 \%$ inactivated fetal calf serum (FCS; EuroClone, Wetherby, UK) and $2 \mathrm{mM} \mathrm{L-glutamine}$ (Sigma-Aldrich, St. Louis, MO, USA). Cells were maintained at $37^{\circ} \mathrm{C}$ in a humidified $5 \% \mathrm{CO}_{2}$ atmosphere.

MTT assay. To test the effects of kinamycin F, osteosarcoma cell lines were cultured for 24 or $48 \mathrm{~h}$ in the presence of the vehicle (DMSO $0.1 \%$ ) or increasing drug concentrations, and cell growth was determined using the MTT (3-(4,5-Dimethylthythiazol-2-yl)2,5-diphenyltetrazolium bromide) cell proliferation kit (Roche Diagnostic, Basel, Switzerland), according to the manufacturer's instructions. Briefly, $0.5 \mathrm{mg} / \mathrm{ml}$ of MTT labeling reagent was added to each well and incubated for $4 \mathrm{~h}$. Purple formazan crystals were solubilized by adding $100 \mu \mathrm{l}$ of the solubilization solution $(0.01 \mathrm{M}$ $\mathrm{HCl}$ and $10 \%$ SDS) overnight. The plate was subsequently read on an Infinite M200 photometer (Tecan Group Ltd., Männedorf, Switzerland) at a wavelength of $570 \mathrm{~nm}$. Colorimetric readings were normalized against plates of non-treated cells under identical culture conditions. The experiment was performed 3 times, each time in triplicate.

Trypan blue dye exclusion assay. The viability effects of kinamycin $\mathrm{F}$ on osteosarcoma cells were determined using trypan blue exclusion and manually counting in microscope chambers. Briefly, cells were washed and suspended $\left(1.0 \times 10^{5}\right.$ cells $\left./ \mathrm{ml}\right)$ in a buffer solution containing PBS 1X, EDTA $0.5 \mathrm{mM}$ (Life technologies, Burlington, ON, Canada), and BSA 0.2\% (Sigma-Aldrich, St. Louis, MO, USA).
Fifty (50) $\mu$ l of cell suspension was taken and mixed with an equal volume of $0.4 \%$ Trypan blue. The solution was mixed thoroughly and allowed to stand for $5 \mathrm{~min}$ at room temperature. $10 \mu \mathrm{l}$ of the solution was transferred to a hemocytometer and viable cells were counted as clear cells and dead cells as blue ones. The number of live cells in both treated and control flasks was used for calculating the percent viability.

Cell cycle analysis. Cell cycle analysis was performed using the Muse $^{\text {TM }}$ Cell Analyzer (Merck Millipore, Milan, Italy). In brief, after $24 \mathrm{~h}$ of treatment, cells were harvested, centrifuged at $300 \times g$ for 5 min and washed once with $1 \mathrm{X}$ PBS. After fixing them with $70 \%$ ethanol for at least $3 \mathrm{~h}$ at $-20^{\circ} \mathrm{C}$, cells were centrifuged at $300 \times g$ for $5 \mathrm{~min}$, washed once with $1 \mathrm{X}$ PBS and then $200 \mu \mathrm{l}$ of Muse $\mathrm{T}^{\mathrm{TM}}$ Cell Cycle reagent was added to each tube with an incubation of 30 min at room temperature in the dark. Samples were then analyzed according to the manufacturer's protocol.

Western blot analysis. Cells were lysed in RIPA lysis buffer containing protease and phosphatase inhibitor cocktails (ThermoFisher Scientific, Waltham, MA, USA), and $25 \mathrm{U} / \mathrm{ml}$ benzonase. The protein concentration was determined by the Bradford Protein Assay (Bio-Rad, Hercules, CA, USA) according to the manufacturer's indications, and $80 \mu \mathrm{g}$ of cellular lysate was separated by SDS-PAGE on $4-20 \%$ gradient gels (ThermoFisher Scientific, Waltham, MA, USA). Gels were transferred onto nitrocellulose membranes and blocked in 5\% BSA in 1× TBS containing $0.1 \%$ Tween-20 (TBST) and then incubated in primary antibody in TBST. Membranes were washed in $1 \times$ PBST and incubated for $1 \mathrm{~h}$ in the appropriate secondary antibody in TBST. Primary antibodies were from Santa Cruz Biotechnology (Santa Cruz, CA, USA): $\alpha$-cyclin A (sc-596), $\alpha$-actin (sc-1616); from Cell Signaling Technologies (Danvers, MA, USA): $\alpha$-cyclin D3 (2936), $\alpha$-Cdk2 (2546), $\alpha$-phospho-Cdk2(Thr160) (2561). The incubation was performed overnight at $4^{\circ} \mathrm{C}$. The blots were washed 3 times with $1 \times$ TBST, detected using SuperSignal West Pico Reagent (Pierce, Rockford, IL, USA), and visualized in a ChemiDoc digital imaging station (Bio-Rad, CA, USA).

Fluorometric caspase-3 enzyme activity assay. Caspase-3 activity in total cell lysates was determined by a fluorometric EnzChek caspase3 assay kit (Molecular Probes, Eugene, OR, USA) using 7-amino-4methylcoumarin-derived substrate Z-DEVD-AMC, according to the manufacturer's instructions. Briefly, kinamycin F and DMSO were administered to the wells $\left(1,5 \times 10^{5}\right.$ cells/well in a 6 -well plate $)$. After $48 \mathrm{~h}$, the cells were harvested using trypsinization and cell lysates were prepared. The protein concentration of the samples was determined using a Bio-Rad protein assay kit (Bio-Rad Laboratories, Hercules, CA, USA) and measured against bovine serum albumin standards. Samples of the cell lysates were seeded into standard black 96 -well plates as each sample contained $50 \mu$ g protein per well. Samples were mixed with reaction buffer and the plate was incubated for $30 \mathrm{~min}$ at room temperature avoiding direct light. Then, $200 \mu \mathrm{mol} / \mathrm{l}$ substrate of caspase-3, Z-DEVD-AMC, was added to each well and incubated for $1 \mathrm{~h}$ at $37^{\circ} \mathrm{C}$ in the dark. The assay conditions were standardized in order that the products of the reaction remained in the linear range of detection. The plate was detected by a fluorescence spectrophotometer using 496/520 nm excitation/emission wavelengths and the sample readings were calculated by subtracting the absorbance of blank samples. 

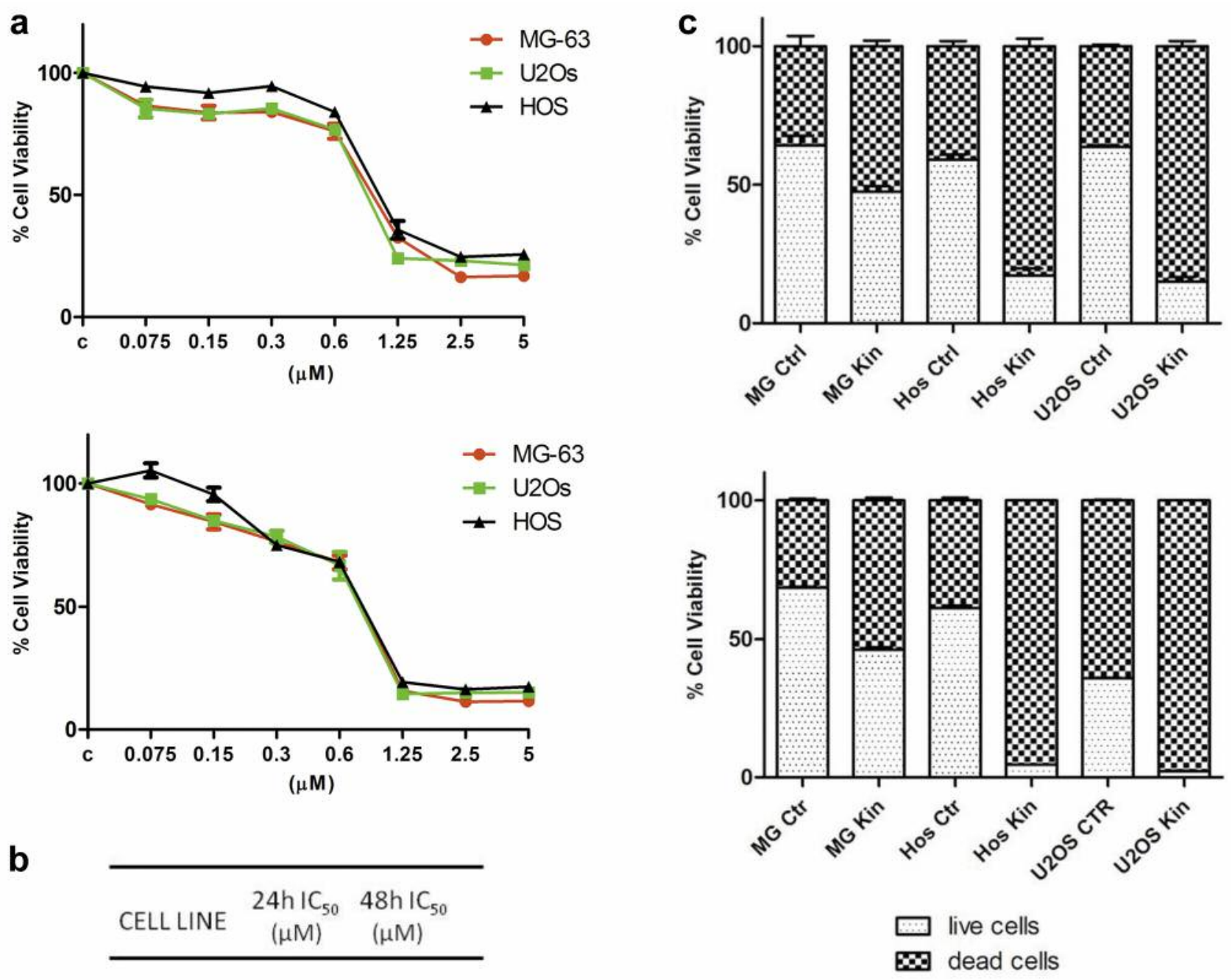

Figure 1. Cell viability rates and $I C_{50}$ s. MTT assays of HOS, MG-63 and U-2 OS treated for 24 and $48 \mathrm{~h}$ with increasing concentrations of kinamycin $F(1 a) . I C_{50}$ s were calculated after 24 and $48 \mathrm{~h}$ of exposure by using the software CalcuSyn $(1 \mathrm{~b})$. Cells were washed and suspended in a buffer solution containing PBS $1 X, E D T A 0.5 \mathrm{mM}$, and BSA 0.2\%. The influence of kinamycin F treatment on the detachment of viable cells grown in culture was studied by comparing the fraction of floating cells between kinamycin F-treated and control cells. Counting and viability were achieved with Trypan blue $0.4 \%$ using a hemocytometer chamber (1c). Results are the mean of three different experiments \pm s.d. Ctrl, Untreated cells.

\section{Results}

Kinamycin $F$ affects cell proliferation and viability in osteosarcoma cell lines. To determine whether kinamycin $\mathrm{F}$ could affect the proliferation and viability of MG-63, U-2 OS and HOS cells were incubated in the presence of increasing concentrations of the drug for either 24 or $48 \mathrm{~h}$ and analyzed by MTT assays. Cell viability rates and $\mathrm{IC}_{50}$ were then calculated. The experiments documented that after $24 \mathrm{~h}$ of treatment, cell proliferation/viability impairment was already evident, with an $\mathrm{IC}_{50}$ value ranging from $0.79 \mu \mathrm{M}$ to $1.03 \mu \mathrm{M}$. After $48 \mathrm{~h}$ of treatment, the impairment was more evident and all tested cell lines demonstrated an appreciable sensitivity to a concentration of $0.7 \mu \mathrm{M}$ (Figures $1 \mathrm{a}$ and $\mathrm{b}$ ). It should be noted that the range in sensitivities among the three cell lines to kinamycin $\mathrm{F}$ was very close and no 


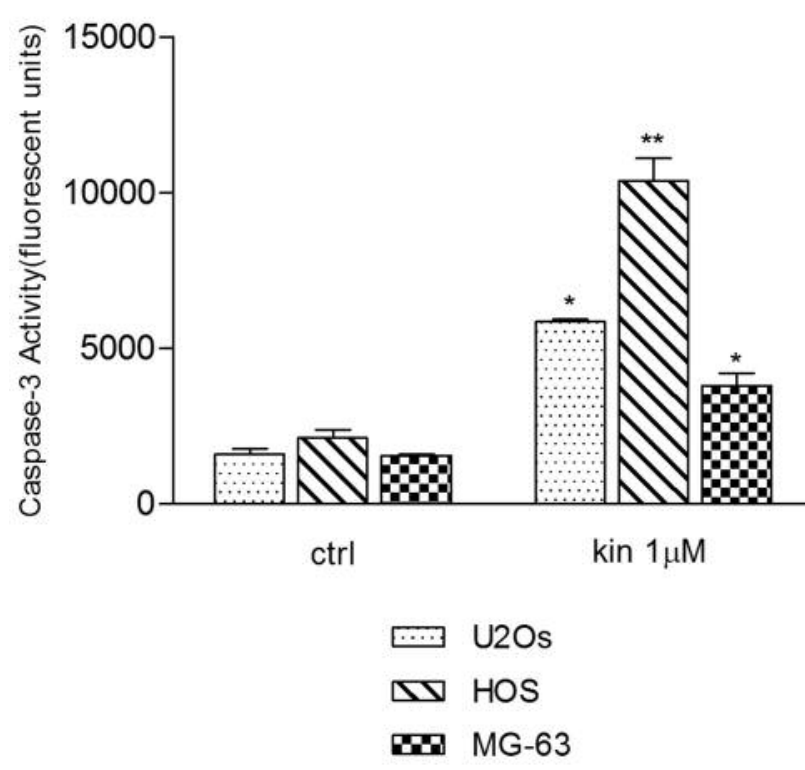

Figure 2. Kanamycin F induced changes in caspase-3 enzyme activity. Osteosarcoma cell protein extracts were analyzed for caspase 3 activity at 24 h of kinamycin F treatment using the caspase substrate Z-DEVD$A M C$ by quantifying fluorescence intensity. Substrate cleavage was measured spectrophotometrically by a fluorescence spectrophotometer using 496/520 nm excitation/emission wavelengths. Twenty-five sample readings were calculated by subtracting the absorbance of blank samples. We observed an increase in caspase-3 activity in all three osteosarcoma cell lines, compared to untreated cells. Statistical analysis was performed using a Student's t-test. ${ }^{*} p<0.05$, ${ }^{*} p<0.01$, vs. control (untreated cells).

significant differences were observable among the cell lines. This observation strengthens the hypothesis that kinamycin $\mathrm{F}$ affected each of these cell lines in a similar way. To confirm the data obtained by MTT assays, the percentage of cell mortality was determined by the Trypan blue assay. We determined the viability and total cell count after a treatment with kinamycin $\mathrm{F}$ for 24 and $48 \mathrm{~h}$. Results obtained after treatment of the three cell lines with $1 \mu \mathrm{M}$ kinamycin $\mathrm{F}$ indicated that the drug induced a concentration-dependent cell death in all the three cell lines (Figure 1c), with a more relevant effect in Hos and U-2 OS than in MG. Data on cellular mortality obtained through Trypan blue analysis confirmed the vitality data obtained in MTT under the same treatment conditions.

Kinamycin $F$ induces apoptosis. Results up to now have suggested that treatment of each of the cell lines in this study with kinamycin $\mathrm{F}$, led to an inhibition of cell proliferation and an increase in mortality. Apoptosis, a regulated form of cell death, is distinguished by unique features; among these is caspase- 3 activation. Caspase- 3 represents the predominant effector caspase responsible for many of the characteristic downstream events associated with apoptosis. Caspases are a family of proteases, subdivided into initiator and effector caspases, involved in initiating and completing apoptotic events. The effector caspases are activated generally by upstream initiator caspases, resulting in a proteolytic cascade which is selfamplifying and irreversible. In order to establish whether decreased cell viability was related to apoptosis in osteosarcoma cell lines, we treated MG, OS and U-2 OS cells for $24 \mathrm{~h}$ with $1 \mu \mathrm{M}$ kinamicin $\mathrm{F}$, and then analyzed caspase-3 activity (Figure 2). After a $24 \mathrm{~h}$ treatment, kinamycin $\mathrm{F}$ was able to activate caspase- 3 in each of the three cell lines, as compared to untreated cells, with a more relevant effect in HOS than in MG-63 and U-2 OS. These results showed that caspase- 3 activity is higher in HOS cells, which present, with respect to other cell lines, a higher mortality at $24 \mathrm{~h}$ of treatment with kinamycin $\mathrm{F}$, as assessed by proliferation and mortality experiments.

Kinamycin $F$ affects cell cycle in osteosarcoma cell line. We also investigated the effects of the drug on cell cycle progression. Fractions of cells in $\mathrm{G}_{0} / \mathrm{G}_{1}, \mathrm{~S}$, and $\mathrm{G}_{2} / \mathrm{M}$-phases were determined after $24 \mathrm{~h}$ of incubation with $1 \mu \mathrm{M}$ kinamycin F. In all cell lines, the analysis showed a significant increase in the $\mathrm{G}_{2} / \mathrm{M}$-phase of the cell cycle (Figure 3). No significant differences appeared between the activity of kinamycin F in MG-63, U-2 OS and HOS cells, as the percentage of cells arrested in the $\mathrm{G}_{2} / \mathrm{M}$-phase remained similar between the cell lines.

Effect of kinamycin F on cyclin A and cyclin D3. The Dtype cyclins are important for progression through the G1phase of the cell cycle as they are responsible for binding and partially activating cyclin dependent kinases, leading to the activation of genes responsible for entry into Sphase. It was already demonstrated that kinamycin $\mathrm{F}$ treatment dramatically reduced cyclin D3 protein levels in K562 cells; thus, the protein levels of cyclin D3 in the osteosarcoma cell lines was examined following treatment. As shown in Figure 4, kinamycin $F$ caused a selective reduction of cyclin D3 protein level in all three osteosarcoma cell lines. These results indicated that cyclin D3 is a potential target of kinamycin $\mathrm{F}$ in osteosarcoma cells as well. Since cells treated with kinamycin F accumulated at the $\mathrm{G}_{2} / \mathrm{M}$ checkpoint, the expression of a key regulatory protein for $\mathrm{G}_{2} / \mathrm{M}$-phase progression, $\mathrm{Cdk} 2$, was evaluated. In MG-63, U-2 OS and HOS cells, the protein expression of cyclin A was increased after kinamycin $\mathrm{F}$ treatment for $48 \mathrm{~h}$. Moreover, the expression of phospho-Cdk2 increased under the same conditions, indicating that kinamycin $\mathrm{F}$ augments the cyclin A-Cdk2 complex in cells arrested in $\mathrm{G}_{2} / \mathrm{M}$. 


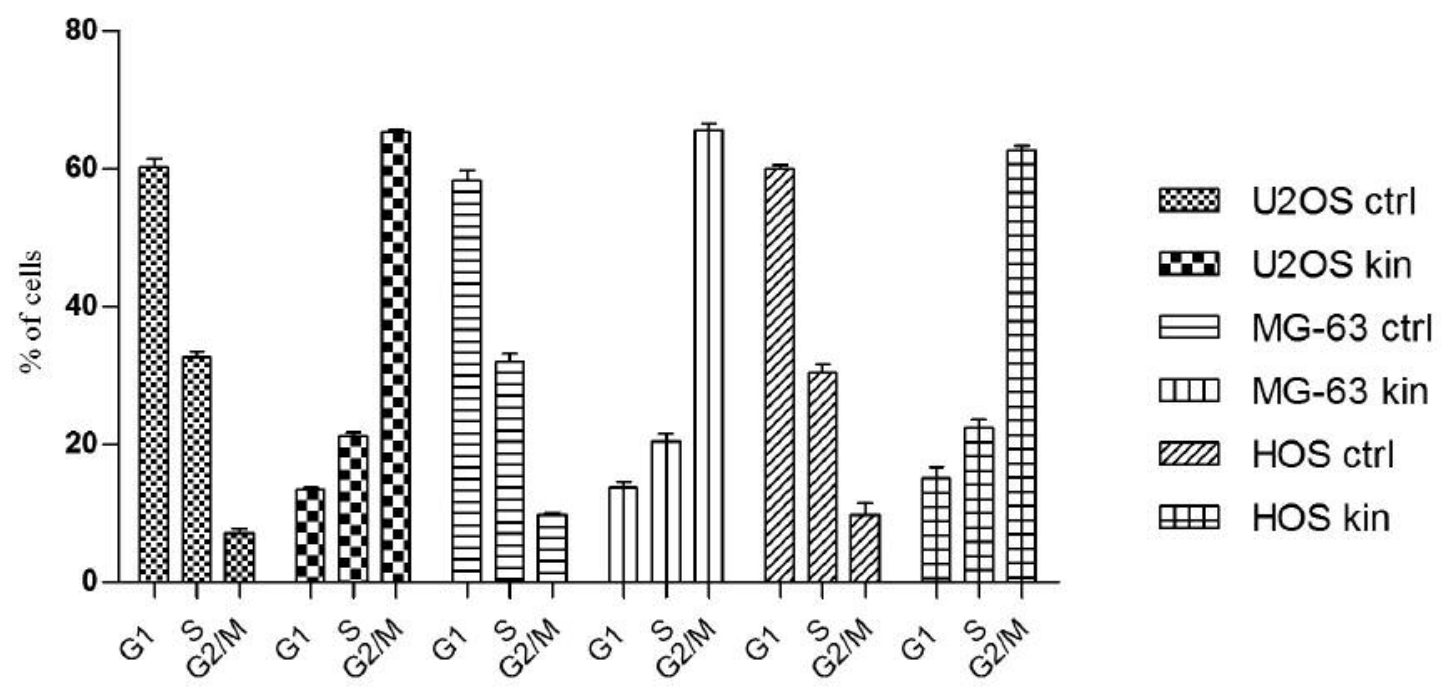

Figure 3. Cell cycle analysis. Analysis of the effects of kinamycin F on cell cycle progression in HOS, MG-63 and U-2 OS were conducted using the flow cytometric Muse ${ }^{T M}$ Cell Analyzer. The indicated data represent mean values $\pm S D$ from $n \geq 3$ independent experiments.

\section{Discussion}

Metastases, tumor recurrences and chemo-resistance are the main causes of treatment failure in osteosarcoma. Therefore, a comprehensive understanding of the mechanisms underlying treatment failure is urgently needed (8). For the therapy of malignant tumors, large cooperative group studies and international collaborations have demonstrated that the most effective regimens include the combination of high-dose methotrexate, doxorubicin, and cisplatin (9). However, malignant osteosarcoma is one of the most multidrug resistant (MDR) malignancies, and thus, many patients are not responsive to chemotherapy (10). As a consequence, these patients have a poor prognosis. Thus, it has become imperative to explore the mechanism(s) underlying the development of osteosarcoma and the associated chemo-resistance.

In recent years, many compounds have been synthesized and tested both in in vitro and in vivo models of different types of cancers $(11,12)$. In the current study, we aimed to investigate the vulnerability of a panel of OS cell lines to the antitumor agent kinamycin F. It has been shown that kinamycin $F$ regulates cell death and induces a differentiating effect on the human erythroleukemia cells K562. In fact, treatment of K562 cells with kinamycin F induced erythroid differentiation, a rapid apoptotic response, induction of caspase-3/7 activities and delayed cell cycle progression through the $\mathrm{S}$ and $\mathrm{G}_{2} / \mathrm{M}$-phases (6). However, its effects had not been previously analyzed in osteosarcoma cells. The findings of this study document that after $24 \mathrm{~h}$ of treatment, cell viability impairment was already observable.

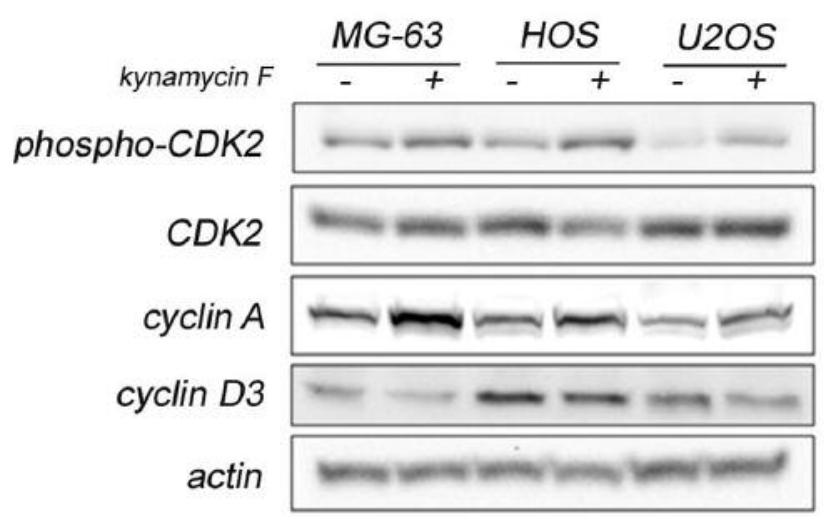

Figure 4. Effect of kinamycin F on cyclins in all cell lines. U-2 OS, M-63 and HOS cell lines were cultured in the presence of kinamycin $F$ $(1,000 \mathrm{nM})$ and analyzed by western blot after $24 \mathrm{~h}$. Cell lysates were loaded at the same volume and protein concentration on a SDS-PAGE $10 \%$ gel, and western blotting analyses were performed with specific antibodies for cyclin A, cyclin D3, Cdk2, p-Cdk2 and actin. Results are representative of three independent experiments. Actin was used as a loading control.

Moreover, an even greater responsiveness of the cells to kinamycin $\mathrm{F}$ was noted after $48 \mathrm{~h}$ of treatment. Our results demonstrated that kinamycin $\mathrm{F}$ suppress osteosarcoma cell proliferation and viability with $\mathrm{IC}_{50}$ values that were comparable at 24 and $48 \mathrm{~h}$. Kinamycin F, besides causing a marked cell growth inhibition, is able to promote apoptosis (7). Treatment of K562 cells with kinamycin F induced erythroid differentiation and a rapid apoptotic response (6). 
In fact, in the present study, we found that kinamycin $\mathrm{F}$ induced apoptosis through caspase- 3 activation in MG-63, U-2 OS and HOS cells. These data suggest the inhibitory effects of kinamycin $\mathrm{F}$ in human osteosarcoma cells. Our data showed that kinamycin $\mathrm{F}$ can suppress cell proliferation, cause $\mathrm{G}_{2} / \mathrm{M}$-phase arrest, and induce apoptosis in human OS cells. The $\mathrm{G}_{2}$ checkpoint prevents cells from entering mitosis when DNA is damaged, providing an opportunity for repair and stopping the proliferation of damaged cells (13). According to flow cytometric analysis, kinamycin $\mathrm{F}$ increased the proportion of cells in $\mathrm{G}_{2} / \mathrm{M}$ and decreased the cell proportion in $\mathrm{G}_{0} / \mathrm{G}_{1}$ - and $\mathrm{S}$-phases in OS cells. Entry into the cell cycle and subsequent checkpoint release at the end of the phases is controlled by the cyclin (cyclin A, B, D and E)-Cdk (Cdk1, 2, 4 and 6) complexes formed. During normal cellular proliferation, cyclin D family members are expressed throughout each phase of the cell cycle and are at their highest during S-phase. In contrast, while cyclin $\mathrm{E}$ is expressed during the $G_{1}-S$ transition and cyclin $B$ is expressed mainly during the $\mathrm{G}_{2} / \mathrm{M}$-phase transition, it is cyclin A which is mostly expressed during the $G_{2}$-phase. Western blotting analysis showed that kinamycin F led to an increase in the accumulation and activation of the $G_{2} / M$ phase-related cycle regulator cyclin A. Furthermore, analysis revealed that kinamycin $\mathrm{F}$ also increased the expression levels of phospho-Cdk2. The complex of Cdk2/cyclin A has a crucial role in promoting the $\mathrm{G}_{2} / \mathrm{M}$-phase transition. Cyclin A associates with $\mathrm{Cdk} 2$ for entry into the $\mathrm{G}_{2}$-phase of the cycle, and this complex must be degraded before the cells can progress to M-phase. The involvement of cyclin A in the $\mathrm{G}_{2} / \mathrm{M}$-phase has been implied in a number of studies, but its precise role and potential substrates remain to be elucidated. Recently it was demonstrated that cyclin A/Cdk2 associates with the centrosomes soon after separation of the centrosome pairs in late $\mathrm{G}_{2}$-phase. Moreover, cyclin $\mathrm{A} / \mathrm{Cdk} 2$ localizes to the centrosome in late $\mathrm{G}_{2}$-phase and coordinates nuclear and centrosomal mitotic events (14). In addition, we examined the effects on cyclin D3. We recently showed that during K562 differentiation process induced by kinamycin F, cyclin D3 level is regulated by PLC $\beta 1$ signaling pathway and in particular the amplification of the expression of the PLC $\beta 1 \mathrm{a}$, but not of PLC $\beta 1 b$, is able to maintain high levels of expression of cyclin D (15). Overexpression of cyclin D is observed in many human tumors. For example, cyclin D3 positive nuclei are present in $62 \%$ of sarcomas, and elevated expression of cyclin D3 has been noted in the human sarcoma cell lines (SKLMS, MG-63, SaOS-2 and HT1080) (16). Interestingly, kinamycin $\mathrm{F}$ treatment resulted in decreased expression of cyclin D3. A similar finding was reported during K562 erythroid differentiation following kinamycin F treatment (6). The loss of cyclin D3 expression together with cyclin A-Cdk2 complex accumulation, causes $\mathrm{G}_{2}$ arrest.
The discovery and development of novel therapeutic targets and strategies against osteosarcoma has remained for the most part elusive. However, in light of the results presented here, kinamycin $\mathrm{F}$ may represent a promising agent for cancer therapy in OS and a better understanding of the mechanism by which kinamycin F exerts its ability to inhibit cell growth may be a step forward in the development of new therapeutic strategies for the treatment of OS.

\section{Conflicts of Interest}

None.

\section{Acknowledgements}

The sample of kinamycin $\mathrm{F}$ that was used in this study was provided by Professor G. I. Dmitrienko at the University of Waterloo, Waterloo, Ontario, Canada.

This work was supported by fundamental contribution of Fondazione del Monte di Bologna e Ravenna to I.F.; by a grant from Associazione Italiana per la Ricerca sul Cancro (AIRC-IG-201517137), to W.B. and a grant "5 per mille" Project 2013/2014 to Rizzoli Orthopedic Institute.

\section{References}

1 Durfee RA, Mohammed M and Luu HH: Review of osteosarcoma and current management. Rheumatol Ther 2: 221-243, 2016.

2 Isakoff MS, Bielack SS, Meltzer $\mathrm{P}$ and Gorlick R: Osteosarcoma: Current treatment and a collaborative pathway to success. J Clin Oncol 33: 3029-3035, 2015.

3 Sakamoto A and Iwamoto Y: Current status and perspectives regarding the treatment of osteo-sarcoma: chemotherapy. Rev Recent Clin Trials 3: 228-231, 2008.

4 Shaikh AB, Li F, Li M, He B, He X, Chen G, Guo B, Li D, Jiang F, Dang L, Zheng S, Liang C, Liu J, Lu C, Liu B, Lu J, Wang L, Lu A and Zhang G: Present advances and future perspectives of molecular targeted therapy for osteosarcoma. Int J Mol Sci 17: 506, 2016.

5 Ta HT, Dass CR, Choong PFM and Dunstan DE: Osteosarcoma treatment: state of the art. Cancer Metastasis Rev 28: 247-263, 2009.

6 O'Hara KA, Dmitrienko GI and Hasinoff BB: Kinamycin F downregulates cyclin D3 in human leukemia K562 cells. Chem Biol Interact 184: 396-402, 2010.

7 O'Hara KA, Wu X, Patel D, Liang H, Yalowich JC, Chen N, Goodfellow V, Adedayo O, Dmitrienko GI, and Hasinoff BB: Mechanism of the cytotoxicity of the diazoparaquinone antitumor antibiotic kinamycin F. Free Radic Biol Med 43: 1132-1144, 2007.

8 Luetke A, Meyers PA, Lewis I and Juergens H: Osteosarcoma treatment - where do we stand? A state of the art review. Cancer Treat Rev 40: 523-532, 2014.

9 Isakoff MS, Bielack SS, Meltzer $\mathrm{P}$ and Gorlick R: Osteosarcoma: Current treatment and a collaborative pathway to success. J Clin Oncol 33: 3029-3035, 2015.

10 Michaelson MD and Smith MR: Bisphosphonates for treatment and prevention of bone metastases. J Clin Oncol 23: 8219-8224, 2005. 
11 Ma Y, Zhu B, Yong L, Song C, Liu X, Yu H, Wang P, Liu Z and Liu X: Regulation of intrinsic and extrinsic apoptotic pathways in osteosarcoma cells following oleandrin treatment. Int J Mol Sci 17: 1950, 2016

12 Zhou C, Tan W, Lv H, Gao F and Sun J: Hypoxia-inducible microRNA-488 regulates apoptosis by targeting Bim in osteosarcoma. Cell Oncol Dordr 39: 463-471, 2016.

13 Stark GR and Taylor WR: Analyzing the $\mathrm{G}_{2} / \mathrm{M}$ checkpoint. Methods Mol Biol 280: 51-82, 2004.

14 De Boer L, Oakes V, Beamish H, Giles N, Stevens F, Somodevilla-Torres M, Desouza C and Gabrielli B: Cyclin A/cdk2 coordinates centrosomal and nuclear mitotic events. Oncogene 27: 4261-4268, 2008.
15 Bavelloni A, Dmitrienko GI, Goodfellow VJ, Ghavami A, Piazzi M, Blalock W, Chiarini F, Cocco L and Faenza I: PLC $\beta 1 \mathrm{a}$ and PLC $\beta 1$ b selective regulation and cyclin D3 modulation reduced by kinamycin F during K562 cell differentiation. J Cell Physiol 230: 587-594,2015.

16 Bui MM, Bagui TK, Boulware DC, Letson DG, Nasir A, Kaiser HE, Pledger WJ and Coppola D: Altered expression of cell cycle regulatory proteins in benign and malignant bone and soft tissue neoplasms. In Vivo 21: 729-737, 2007.

Received June 7, 2017

Revised June 25, 2017

Accepted June 26, 2017 